\title{
Management of Atypical Suicidal Cut Throat Injury in a Private Tertiary Hospital
}

\author{
Adeyeye Rachael Adetola ${ }^{1}$, Akinola Moses Ayodele ${ }^{1}$, Fasesan Oluwatoyin ${ }^{2}$, Ogundare Emmanuel ${ }^{3}$, \\ Agbaakin Adewale Daniel ${ }^{1}$
}

${ }^{1}$ Otorhinolaryngology Unit, Department of Surgery, Babcock University Teaching Hospital, Ilishan Remo, Nigeria

${ }^{2}$ Psychiatry Unit, Department of Medicine, Babcock University Teaching Hospital, Ilishan Remo, Nigeria

${ }^{3}$ Anesthesiology Unit, Department of Surgery, Babcock University Teaching Hospital, Ilishan Remo, Nigeria

Email address:

akinolam $@$ babcock.edu.ng (A. M. Ayodele)

\section{To cite this article:}

Adeyeye Rachael Adetola, Akinola Moses Ayodele Fasesan Oluwatoyin, Ogundare Emmanuel, Agbaakin Adewale Daniel. Management of Atypical Suicidal Cut Throat Injury in a Private Tertiary Hospital. International Journal of Otorhinolaryngology. Vol. 5, No. 1, 2019, pp. 5-8. doi: $10.11648 /$ j.ijo.20190501.12

Received: December 31, 2018; Accepted: January 22, 2019; Published: February 19, 2019

\begin{abstract}
Cut throat injuries are potentially life threatening due to the peculiarity of the structures traversing this region of the body. These injuries could be accidental, homicidal or suicidal and the use of a sharp weapon is the least frequent suicidal method in most cases reported. In cases of attempted suicide, hesitant marks are a common finding. Attempting suicide by cutting the throat without hesitant marks is a very rare occurrence. Generally, cases of cut throat injuries are largely under reported as many are fatal before presenting to the hospital therefore a definite incidence is not available. These patients need emergency multidisciplinary care to prevent rapid deterioration, avoidable morbidity and mortality. Adequate airway management skill in intubating and securing the airway to allow for wound exploration and surgical repair is essential for a good outcome. We present to you a rare case of suicidal cut throat injury in a 38 year old male with depressive type of schizoaffective disorder not associated with hesitant marks at Babcock University Teaching Hospital, a private tertiary hospital in south west Nigeria. The rarity of the case is the reason for presenting this case report.
\end{abstract}

Keywords: Suicidal Cut Throat Injuries, Incidence, Morbidity, Mortality, Hesitant Marks

\section{Introduction}

Suicide is a major cause of mortality worldwide. It is a human tragedy that accounts for an estimated one million deaths annually [1]. The incidence of attempted suicide is about twenty times more than that of completed suicide [2]. There are several methods of attempting suicides. According to some reports from studies done in Nigeria, the most popular methods of attempting suicide includes hanging, use of firearms and ingestion of poisonous substances in that order. [3] Throat cutting remains a rare method [4]. More rare is attempting suicide by throat cutting without hesitant marks as seen in the case report[5]. Several risk factors have been implicated in suicide and attempted suicide and some of these includes mental illnesses, physical health problems, drug and alcohol abuse, interpersonal difficulties as well as socioeconomic problems among others. [6] WHO mortality base shows that 85 per cent of the world's suicides occur in low and middle income countries. [7] The prevalence of attempted suicide in Nigeria is 0.7 per cent. [8] When suicidal cut throat injuries occur, a multidisciplinary approach is required in the effective management of the victim and requires the close collaboration of the otorhinolaryngologist, anesthesiologist and the psychiatrist.

The diagnosis is based on history and examination of the pharynx, larynx, oesophagus and other contiguous structures to determine the extent of the injury. The neck accommodates vital structures like neurovascular bundles, larynx, trachea and oesophagus. Injuries to these structures are varied and depend on the site, pattern and depth of the cut on the neck.

\section{Case Report}

Patient is a 38 year old single unemployed male of Yoruba ethnicity who was being managed for severe depressive 
illness at a neuropsychiatric hospital with poor medication compliance since two years prior to presentation. $\mathrm{He}$ attempted suicide by slitting his throat with a razor.

Patient was found in his room in the pool of his blood with a blood stained razor in his right hand. Family members present rushed him to a general hospital where first aid treatment and anti-tetanus prophylaxis was given before referral to our facility. He was brought to our emergency unit with a wide open gaping wound on the anterior neck at the level of the cricoid cartilage. A roll of gauze was used to pack the wound, it was soaked with blood and bubbles of air were seen on it. He was breathing through the retracted end of the larynx evidenced by the gush of expiratory air coming out of it. He presented with cough, dyspnea, aphonia, breathlessness and tachypnea. He had a pulse rate of 120 per min, blood pressure of $90 / 60 \mathrm{mmHg}$ and respiratory rate of 32 per minute with oxygen saturation between 68 and 72 percent. He had coarse pulmonary crepitations in the middle and lower lung zones bilaterally. At the emergency room, two wide bore cannulas were placed, volume resuscitation was carried out with two units normal saline. Blood sample was taken for urgent Packed Cell Volume (PCV), blood grouping and cross matching. High risk informed consent was obtained and patient was admitted under ASA IV for emergency surgery.

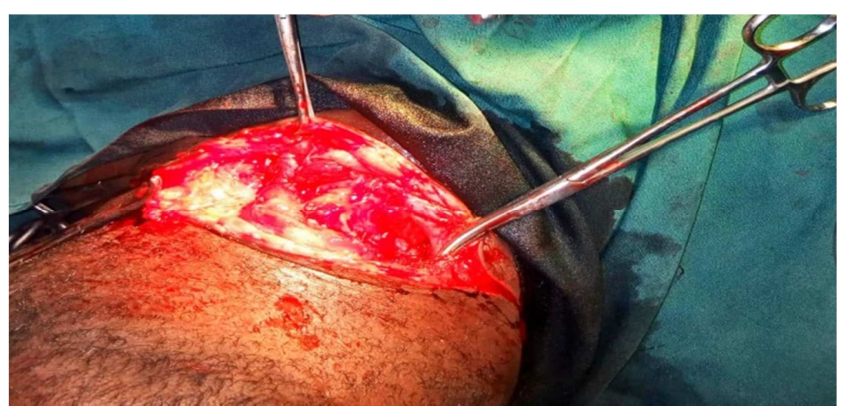

Figure 1. Patient in the theatre before intubation

In the operating theatre, continuous electrocardiogram, non-invasive blood pressure and pulse-oximeter monitoring were carried out. (Heart rate was 80 beats per minute, blood pressure was $140 / 80 \mathrm{mmHg}$, and saturation ranged between $74 \%$ and $85 \%$ (on oxygen by face mask)).

Patient was positioned supine and premedicated with intravenous atropine $0.6 \mathrm{mg}$, metoclopramide $10 \mathrm{mg}$ and midazolam $1 \mathrm{mg}$. Induction was carried out with intravenous ketamine $100 \mathrm{mg}$, using pethidine $1 \mathrm{mg} / \mathrm{kg}$ body weight for analgesia while atracurium was used for muscle relaxation, maintained with oxygen and isoflurane. Attempted intubation with a size $7.0 \mathrm{~mm}$ endotracheal tube through the oropharynx failed as a result of the disconnection from the laceration preventing adequate exposure into the larynx to secure the airway.

The size $7.0 \mathrm{~mm}$ cuffed endotracheal tube was routed through the wound, checked and position confirmed. With Magill's forceps, the endotracheal tube was pulled on the proximal end through the wound via the oropharynx and into the oral cavity and airway was secured. Anaesthesia was maintained with oxygen, isoflurane and intravenous atracurium as muscle relaxant. Monitoring with non-invasive blood pressure, oxygen saturation, heart rate, Electrocardiogram, end tidal carbon dioxide, temperature and urine output were done.

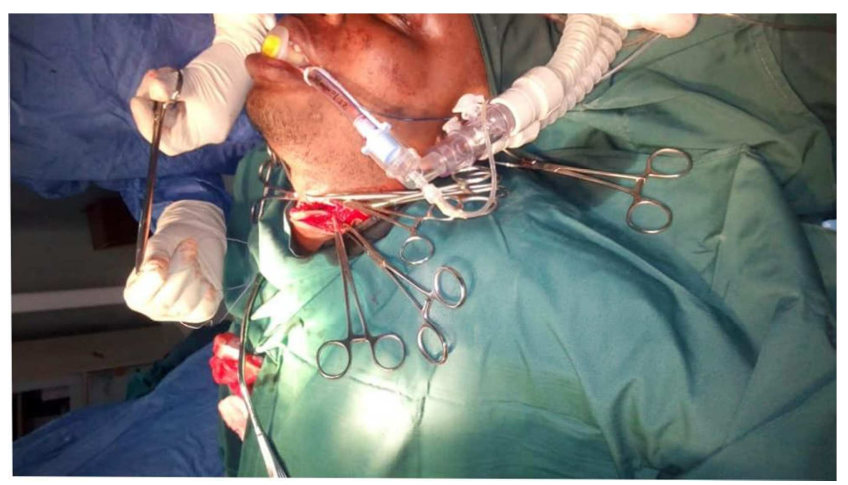

Figure 2. Patient in the theatre after intubation.

With the patient maintained in the supine position, the neck extended using a shoulder roll to adequately expose the wound site. The head was stabilized with a head ring. Appropriate scrubbing and draping was done. Exploration of the wound revealed a transverse laceration to the anterior neck over the region of the cricoid cartilage. It was $7 \mathrm{~cm}$ below the mandible and $7 \mathrm{~cm}$ above the suprasternal notch. Left lateral end was $4.5 \mathrm{~cm}$ below the tip of the left mastoid while the right lateral end was $5 \mathrm{~cm}$ below the tip of the right mastoid. The laceration was $2.5 \mathrm{~cm}$ deep on the left lateral end and $1.5 \mathrm{~cm}$ deep on the right lateral end (this can be explained by patient's right handedness) and measured $12 \mathrm{~cm}$ in length spanning through the medial borders of both sternocleidomastoid muscles. The laceration was a single transverse one with no hesitant marks. Angles of the injury at both ends were acute. It traversed the skin, subcutaneous tissue, superficial fascia, platysma muscle, strap muscles, pretracheal fascia and the anterior wall of the larynx in the region of the cricoid cartilage about $2 \mathrm{~cm}$ below the laryngeal prominence. The laryngeal lumen was communicating with the exterior via the wound. The major blood vessels of the neck (internal and external carotid arteries and internal and external jugular veins) were spared. Wound site was debrided and devitalized tissues including small fragments of cartilages were carefully removed.

The surgical repair of the transected larynx was first carried out using 3.0 vicryl via simple interrupted suturing technique. The strap muscles were repaired layer by layer via a similar technique using 3.0 vicryl. The undersurface of the skin was under-run with the muscle fascia to reduce dead spaces which could lead to haematoma formation and subsequent infection with 3.0 vicryl. Skin edges were apposed with nylon 2.0 using simple interrupted technique. Wound was subsequently cleaned and dressed. Patient lost about $400 \mathrm{mls}$ of blood (intraoperative loss and blood suctioned from the airway). He was transfused with one unit of whole blood intraoperatively. 


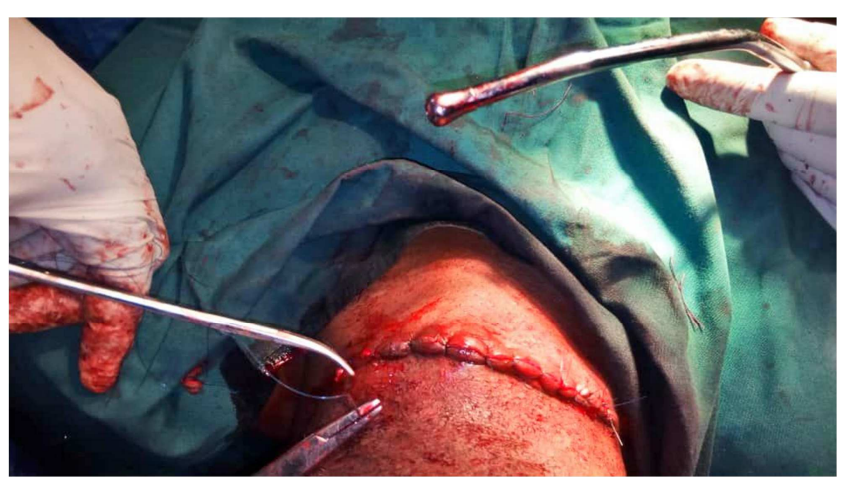

Figure 3. Patient in the theatre after completion of repair.

\section{Results}

On completion of the surgery, patient was transferred to the ICU where anaesthesia was reversed with endotracheal tube in situ and oxygen provided at the rate of $4 \mathrm{~L} / \mathrm{min}$ with oxygen saturation maintained at 95-99 percent. Patient was placed on antibiotics and steroid (IV dexamethasone for 24 hours). Post-operative pain was adequately managed with analgesics. Patient responded very well and was extubated on the second post-operative day and commenced graded oral feeding afterwards which was well tolerated. He was assessed by the psychiatrist on the second post-operative day and the review revealed that the act was premeditated with previous behavioral changes like withdrawal to self and reduced appetite. He attested to auditory hallucinations and reduced mood, reduced energy and loss of interest in previously pleasurable activities. An assessment of depressive type of schizoaffective disorder was made. All sharp and potentially harmful objects were removed from his bed side and nurses and family members were always at his bed side to monitor him. He was commenced on antidepressants and anti psychotics. He also had individual psychotherapy sessions with the clinical psychologist. Over the period of a week, patient's speech and appetite improved, delusions began resolving and he became remorseful about attempting suicide.

The nylon sutures were removed on the seventh postoperative day and patient was discharged from the hospital on the eight post-operative day with residual hoarseness for follow up at the ENT clinic. Patient presented at our clinic two weeks after discharge (three weeks after the incident) for follow up. Anterior neck wound had healed leaving a transverse scar. Residual hoarseness persisted. Fibreoptic laryngoscopy done showed grade 1 glottic and sub glottic stenosis (see figure 5). Patient became compliant with his antidepressant and antipsychotic medications and was subsequently referred back to the neuropsychiatric hospital for continued psychiatric care based on care givers request and proximity and has been regular with his clinic visits. $\mathrm{He}$ is still being followed up in the ENT Clinic of Babcock University Teaching Hospital.

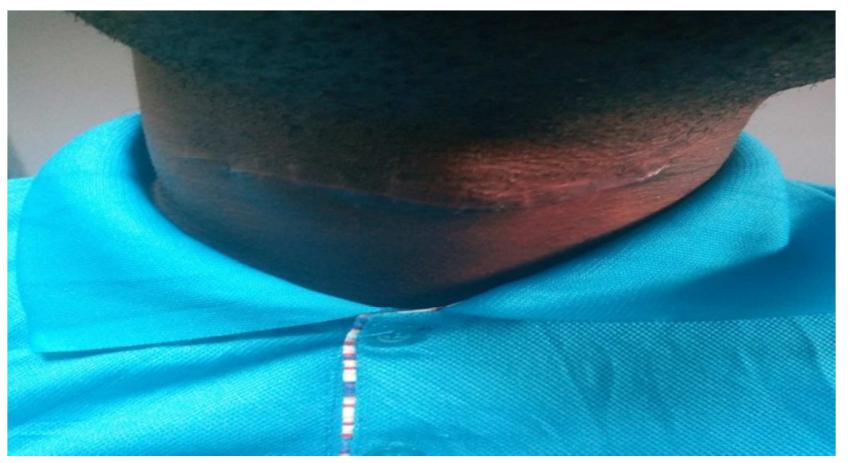

Figure 4. Patient at follow up two weeks after repair.

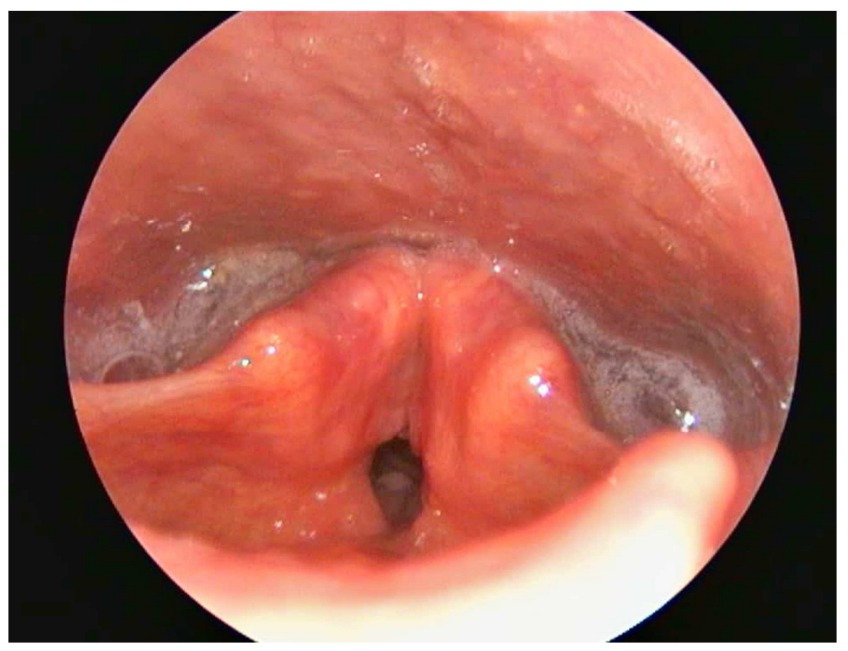

Figure 5. Fibreoptic laryngoscopy done three weeks after repair.

\section{Discussion}

The incidence of suicidal cut throat injuries in our country may be fortunately rare or grossly under reported in literature.

However, when they occur, a multidisciplinary management between the otorhinolaryngologist, the anesthetist and the psychiatrist is required. Assessment of these patients begins with the ABCs of resuscitation.

Our patient presented in respiratory distress and the management of the airway was via the use of endotracheal tube to secure a reliable airway and through which anesthetic gases were administered to allow for proper surgical repair of the lacerated anterior neck structures under general anaesthesia.

The anesthesiologist secured an intact airway, the otorhinolaryngologist explored the wound, assessed the injury and surgically repaired it to restore breathing, swallowing and phonation while the psychiatrist managed the underlying depressive illness.

Suicide is one of the ten leading causes of death with more than a million deaths occurring annually [1]. It is about 20 times more common in individuals with major depressive illness than in the general populace and as such, these patients will require psychiatric evaluation and intervention [9] There is a need for close psychiatric care and supervision in the immediate post- operative period and discharge from 
otorhinolaryngologist care.

Unemployment and relationship breakdown are stressful life events that can lead to suicide as seen in the index case. Previous studies have demonstrated an increase in the rates of suicide and attempted suicide among unemployed individuals than in the general population [10] There is a bidirectional relationship between mental illness and unemployment as individuals with mental illness are less likely to be employed than those without mental illness and the lack of employment in those with mental illness can aggravate the illness [11] The need to screen the general populace for mental illness and adequately treat affected individuals cannot be overemphasized. Since about $85 \%$ of all cases of suicide occur in the middle and low income countries, improvement in the socioeconomic status of the populace will translate into a reduction in the rate of suicide and attempted suicide.

\section{Conclusion}

Suicidal cut throat injuries though seen in our environment are rare.

A close collaboration between the otorhinolaryngologist, anesthetist and the psychiatrist is essential for a favourable outcome.

Adequate screening for mental illness and provision of adequate psychiatric care is essential for preventing suicide and parasuicide including suicidal cut throat injuries.

\section{Competing Interests}

All the authors do not have any possible conflicts of interest.

\section{Author's Contributions}

i. Adeyeye Rachael Adetola: literature review, writing of otolaryngological aspect of case report and discussion.

ii. Akinola Michael Ayodele: proof reading and editing of manuscript.

iii. Fasesan Oluwatoyin: writing of the psychiatry aspect of case report.

iv. Ogundare Emmanuel: writing of the anaesthesia aspect of case report

v. Agbaakin Adewale Daniel: Otolaryngological aspect of case report

\section{References}

[1] Rajiv Radhakrishnan. Chittaranjan Andrade, Suicide: an Indian perspective, indian journal of psychiatry.2012Oct- Dec; 54 (4): 304-319 doi: 0.4103/0019-5545.104793M.

[2] Suicide Prevention (SUPRE)

[http:/www.who.int/mental_health/prevention/suicide/suicidep revent/en/] [Ref list].

[3] SAU Offiah, CC Obiorah, Pattern of suicide in Nigeria: the Niger Delta experience, jourmal of medical investigations and practice 2014, volume 9 issue 1 page8-11 doi: $10.4103 / 9783 \backslash 1230.13255$.

[4] S. Fukube, T. Hayashi, Y. Ishida, H. Kamon, M. Kawaguchi, A. Kimura et al. Retrospective study on suicidal cases by sharp force injuries. J Forensic Leg Med 15 (2008), pp 163 167.

[5] B Ozdemir, O Celbis, A Kaya Cut throat injuries and honor killings: review of cases in eastern Turkey. J Forensic Leg Med, 20 (4) (2013), pp. 1198-203.

[6] Becky Mars, Stephanie Burrows, Heidi Hjelmeland, David Gunnell. Suicidal behaviour across the African continent: a review of the literature. BMC Public Health.2014; 14: 606 doi:10.1186/1471-2458-14-606.

[7] Krug EG, Dahlberg TT, Mercy JA, Zwi AB, Lozano R. World report on violence and health. 2002. World Health Organization, Geneva.

[8] Gureje O, Kola L, Ukakwe R, Udofia O, Wakil A, Afolabi E. The profile and risks of suicidal behaviours in the Nigerian Survey of Mental Health and Well-Being. Psychol Med. 2007; 37 (6): 821-830. doi: 0.1017/S0033291707000311.[PubMed][Ref list].

[9] Terra JL. Suicide risk and depression. Rev Prat 2008, 58 (4): 385-8.

[10] Platt S: Unemployment and suicidal behavior: a review of the literature. Soc Sci Med 198419 (2): 93-115.

[11] Adoga et al: Management of suicidal cut throat injuries in a developing nation: three case reports. Cases journal 2010 $3: 65$. 